\title{
Expertengespräch: Chronische Erkrankungen und Multimorbidität - Teil 1
}

Mit Homöopathie bzw. Funktioneller Biometrie, Chinesischer Medizin und Akupunktur lassen sich chronische Erkrankungen gut behandeln. Dr. Beat Hornstein behandelt seine Patienten konsequent nach diesem Ansatz.

Welche chronischen Beschwerden sehen Sie am häufigsten in Ihrer Praxis?

Dr. Beat Hornstein: Ich habe viele verschiedene Gebiete, die ich mit der Traditionellen Chinesischen Medizin, mit der Homöopathie und mit der Akupunktur abdecke. Ein grosses Feld sind die atopischen und allergischen Erkrankungen wie Neurodermitis, Pollinose oder Asthma Bronchiale sowie weitere Hauterkrankungen wie Urtikaria und Akne vulgaris. Deshalb behandle ich wahrscheinlich auch viele Säuglinge und Kinder; die Jüngsten kommen schon kurz nach der Geburt. Häufig sind auch neurologische Erkrankungen, z.B. chronische Schmerzsyndrome, Kopfschmerzen und Neuralgien, aber auch Multiple Sklerose und chronische Müdigkeitssyndrome. Die älteren Patienten kommen hauptsächlich mit Beschwerden aus dem rheumatischen Formenkreis, mit Polyarthrosen, Polyarthritiden und anderen rheumatischen Erkrankungen. Patienten mit Krebs und Depressionen suchen auch Hilfe in der Komplementärmedizin. Speziell häufig sind Patienten mit eher seltenen Erkrankungen oder Erkrankungen mit hartnäckigem Verlauf oder Therapieresistenz: die «schwierigen Fälle».
Was ist die Motivation der Patienten, für eine Behandlung von chronischen Erkrankungen ganzheitliche Methoden zu versuchen?

Das ist sehr unterschiedlich. Ein grosser Teil war schon in schulmedizinischer Behandlung und sucht bewusst neue Wege, möchte eine Alternative nach dem Motto «weg von der Chemie». Viele Patienten kommen aber auch einfach, weil sie sich Linderung oder Heilung erhoffen - unabhängig von der Methode und egal, ob ganzheitlich oder nicht. Nicht wenige waren vor Jahren bei meiner Praxis-Vorgängerin in Behandlung und machten gute Erfahrungen, sodass sie später wieder kommen.

Wie geht Ihr Ansatz über das Spektrum der herkömmlichen Chinesischen Medizin, Homöopathie und Akupunktur hinaus?

Es ist ein Mosaik, in dem die Chinesische Medizin bzw. das Verständnis der Chinesischen Medizin neben der Akupunktur und der Homöopathie eine grosse Rolle spielt. Hinzu kommt noch die sogenannte Elektroakupunktur nach Dr. Voll (EAV), die von meiner Vorgängerin Frau Dr. E. Studer praktiziert wurde. Sie hat versucht, die ursprünglich etwas komplizierte und aufwendige EAVMethode zu vereinfachen, und schuf damit die «Funktionelle Biometrie». Mit der Funktionellen Biometrie kann ich sowohl gewisse Krankheiten und Krankheitsanlagen feststellen als auch die dafür geeigneten Medikamente und Heilmittel. Dabei werden mittels eines Griffels und einer Elektrode an den Akupunkturpunkten der Hand Messungen vorgenommen, wobei sich Medikamente oder Nosoden (homöopathisch verdünnte Krankheitsextrakte) im Messkreis befinden. Der Vorteil dieser Methode liegt in der direkten Austestung einer möglichst Erfolg versprechenden Therapie am Patient.

Man findet schneller eine Therapie, die Erfolg verspricht?

Aus meiner Erfahrung geht das sehr gut. Ich kann auch schulmedizinische Medikamente miteinbeziehen. Das ist ein Vorteil: Wenn ein Patient etwa gewisse Antihypertensiva (Blutdruckmittel) nicht verträgt, kann ich ihm eine Alternative anbieten und ihm zwei, drei andere Medikamente empfehlen. Das Hauptgewicht liegt jedoch bei den homöopathischen Heilmitteln und den Phytotherapeutika. 


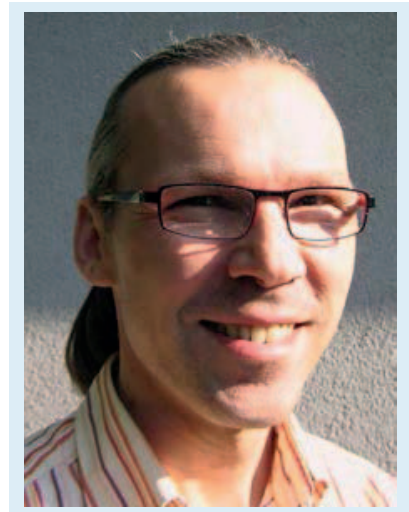

Dr. Beat Hornstein

(beat.hornstein@hin.ch) führt eine Praxis in Basel und hat sich auf Homöopathie, Chinesische Medizin und Akupunktur spezialisiert. Im Vorstand der Union schweizerischer komplementärmedizinischer Ärzteorganisationen hat er lange Zeit die Assoziation Schweizer Aerztegesellschaften für Akupunktur und Chinesische Medizin (ASA) vertreten.

«Der komplementärmedizinische Ansatz kann nur dann erfolgreich sein, wenn man auch die schulmedizinischen Grundlagen mitbringt.»

Spielt die individualisierte, massgeschneiderte Medizin bei der Behandlung chronischer Erkrankungen eine besondere Rolle?

Die massgeschneiderte, individualisierte Medizin ist bei vielen Patienten mit chronischen Erkrankungen wichtig, weil diese eher eine Alternative suchen, als wenn nur eine vorübergehende Erkrankung vorliegt, die sich auch mit schulmedizinischen Methoden gut behandeln lässt. Es ist aber auch so, dass gemäss ganzheitlichem Krankheitsverständnis unterschiedliche Ursachen und Krankheitsbedingungen $\mathrm{zu}$ schulmedizinisch identischen Diagnosen führen. Aufgrund der unterschiedlichen Krankheitsentstehung z.B. einer Colitis ulcerosa (Dickdarmentzündung) ergibt sich jeweils eine ganz andere Therapie.

Interessanterweise zeigen neuere Forschungsergebnisse, dass die Ver- stoffwechslung von Medikamenten sehr unterschiedlich und individuell ablaufen kann. Dies unterstützt den Ansatz einer individuell angesetzten Therapie.

\section{Wie würden Sie die Neurodermitis behandeln?}

Wie oben erwähnt auch individuell, weil eine «Neurodermitis» nicht gleich «Neurodermitis» ist. Bei Hauterkrankungen mit Juckreiz und Ausschlag stehen uns grundsätzlich sehr viele homöopathische und phytotherapeutische Heilmittel zur Verfügung. Die Austestung mittels Funktioneller Therapie kann deshalb manchmal zeitaufwendig sein. Oft kann aber eine Eingrenzung aufgrund der Vorgeschichte, der körperlichen Merkmale und der Verhaltensweisen vorgenommen werden, sodass sich der Aufwand im Rahmen hält. Neben der Verabreichung von individuellen Heilmitteln spielen die Ernährung und die Lebensführung eine grosse Rolle. Hier erfolgt eine entsprechende Beratung mit individuellen Empfehlungen.

Wie fällen Sie die Entscheidung, ob Sie Neurodermitis homöopathisch oder mit Akupunktur behandeln?

Bei Kindern und Jugendlichen praktiziere ich selten Akupunktur. Man könnte sie zwar akupunktieren, z.B. auch Kleinkinder mittels «QuickNeedling», aber oft besteht eine Nadelphobie. Bei Kindern zeigen homöopathische Heilmittel bei der Neurodermitisbehandlung mithin die besten Erfolge. Phytotherapeutische Mittel kann man zusätzlich bei Jugendlichen ab ca. 8-10 Jahren einsetzen. Bei Erwachsenen beginne ich meistens mit einer homöopathischen Behandlung und entscheide dann im weiteren Verlauf, ob eine zusätzliche Akupunkturbehandlung indiziert ist.
Oft ist eine Akupunkturbehandlung nicht notwendig.

Viele Kinder und Jugendliche sind wegen ADHS in Behandlung. Bietet Ihnen die Homöopathie Aussicht auf einen Behandlungserfolg?

Ich habe eine ganze Reihe von Kindern als Patienten, die wegen dieser Diagnose zu mir kommen. Der Erfolg der Behandlung ist sehr gut - allerdings muss man sich vor Augen halten, dass sich die Behandlung über mehrere Jahre hinziehen kann. Das erfordert dann von allen Beteiligten Geduld.

Braucht es zusätzlich noch eine Art von Verhaltenstherapie oder kann man ADHS homöopathisch in den Griff bekommen?

Es gibt Patienten, die auf eine alleinige homöopathische Behandlung relativ rasch und anhaltend ansprechen. Dann erübrigt sich eine Verhaltenstherapie oder eine medikamentöse Therapie mit Ritalin.

Bei längerer Behandlung und ausgeprägtem ADHS kann eine psychotherapeutische Behandlung notwendig sein. In meiner Praxis sehe ich häufig, dass eine «normale» empathische Zuwendung zum Patienten neben der homöopathischen Therapie ausreichend ist.

Wie behandeln Sie ältere Patienten, die etwa mit Gicht zu Ihnen kommen?

Grundsätzlich besteht das gleiche individuelle Vorgehen wie oben beschrieben. Bei älteren Patienten mit längerer Vorgeschichte braucht es mehr Zeit, bis sie auf die Therapie ansprechen. Ich erlebe häufig, dass man die Medikamente schrittweise reduzieren kann - ersetzen kann 
man sie nicht immer. Dann bilden Homöopathie, Akupunktur und Chinesische Medizin eine echte komplementäre Therapie zur konventionellen Medizin.

Kann man eine beginnende Gicht homöopathisch oder mit der Akupunktur in den Griff bekommen?

Bei der Gicht spielen die Lebensweise und die Ernährung eine grosse Rolle. Daher reicht eine alleinige homöo- pathische Behandlung oder Akupunktur auf Dauer nicht aus. Im Schub mildern beide Methoden zwar die Beschwerden, aber für einen anhaltenden Erfolg ist eine Verhaltensänderung notwendig.

Welche Rolle spielt die Schulmedizin für Sie bei der Behandlung chronischer Erkrankungen?

Die Kausalitäten hinter den Krankheiten $\mathrm{zu}$ erkennen und $\mathrm{zu}$ ver- stehen, ist für mich enorm wichtig. Zudem müssen die bisher erfolgten Therapien, die die Patienten bekommen haben, der aktuelle Gesundheitszustand und mögliche Krankheitsverläufe umfassend beurteilt werden können. Dazu ist eine gründliche schulmedizinische Ausbildung nötig.

Der komplementärmedizinische Ansatz kann nur dann nachhaltig erfolgreich sein, wenn man auch die schulmedizinischen Grundlagen mitbringt.

Interview: Oliver Klaffke 